\title{
Application of Environmental Energy as Power Supply of Wireless Sensor along DC Electric Railway
}

\author{
Kazuhiko Ito, ${ }^{1 *}$ Hirokazu Miyaguchi, ${ }^{1}$ Kazuhiro Yamamoto, ${ }^{2}$ \\ Masahiko Ito, ${ }^{3}$ Hideki Masudaya, ${ }^{4}$ and Yoshinobu Maeda ${ }^{5}$ \\ ${ }^{1}$ Electrical Technology Research Office, West Japan Railway Company, \\ 2-4-24 Shibata, Kita-ku, Osaka City, Osaka 530-8341, Japan \\ ${ }^{2}$ New Business Development Center, Fujikura Ltd., \\ 1440 Mutsuzaki, Sakura-shi, Chiba 285-8550, Japan \\ ${ }^{3}$ Materials Science Research Laboratory, Central Research Institute of Electric Power Industry, \\ 2-6-1 Nagasaka, Yokosuka-shi, Kanagawa-ken 240-0196, Japan \\ ${ }^{4}$ Engineering Headquarters Furukawa R\&D Center, ALPS ALPINE Co., Ltd., \\ 6-3-36 Nakazato Furukawa, Oosaki, Miyagi 989-6181, Japan \\ ${ }^{5}$ Department of Electric and Electronic Engineering, School of Science and Engineering, Kindai University, \\ 3-4-1 Kowakae, Higashiosaka City, Osaka 577-8502, Japan
}

(Received October 4, 2019; accepted November 5, 2019)

Keywords: energy harvesting, electric railway, wireless sensor

Assuming that a wireless sensor is installed to determine the state of facilities along a railway, we verified the possibility of applying environmental energy as a power source for the sensor. We measured different types of environmental energy and calculated the potential electric energy that can be generated from each type. The application of different methods of power generation to a wireless sensor power supply was studied on the basis of the calculated amount of power generated. As a result, we found that solar power generation has sufficient potential for this purpose. We also found that power generation methods such as vibration power generation and magnetic field power generation can be applied according to the installation location, the frequency of measurement/communication, and the data collection method used.

\section{Introduction}

In recent years, wireless sensor modules have been actively developed for monitoring the state of facilities of public infrastructure such as roads and railways. Railway companies are researching the use of wireless sensors to monitor equipment, and these sensors use a combination of photovoltaic power and batteries. ${ }^{(1-3)}$ Along with the expected decrease in the working population of Japan, there is a movement to replace inspection by humans with inspection by sensors and to move from time-based maintenance (TBM) to condition-based maintenance (CBM). For this reason, it is expected that opportunities to use wireless sensors to monitor facilities along railway lines will increase.

*Corresponding author: e-mail: kazuhiko-itou2@westjr.co.jp https://doi.org/10.18494/SAM.2019.2647 
The low-voltage power source necessary for these sensors is produced by stepping down the voltage from the high-voltage distribution line laid along the railway using a transformer. Transformers are not installed at all locations along the track, and the construction of new transformers has the problems of introduction costs and increasing the number of facilities requiring inspection. For this reason, research on energy harvesting to extract electric power from minute amounts of environmental energy has been carried out, and research on the use of electric wire vibration is also being carried out. ${ }^{(4)}$ Although it is considered that a large amount of environmental energy is present along a railway in addition to the vibration, the amount of power that can be obtained is not clear.

In this study, we determined the potential electric energy of various types of environmental energy generated along a railway and verify the possibility of its application to wireless sensors. First, in Sect. 2, the energy sources that can be obtained along the DC electric railway are organized. Next, Sect. 3 shows the measurement contents and results of energy sources selected from the energy sources organized in Sect. 2. Section 4 shows the results of power generation calculated using the measurement results obtained in Sect. 3. Finally, in Sect. 5, the possibility of applying environmental energy as a power supply of a wireless sensor based on the power generation calculated in Sect. 4 is evaluated.

\section{Energy Sources along a Railway}

The following types of environmental energy are generated along a railway. Light energy can be obtained from sunlight, railroad crossings, stations, lighting equipment in tunnels, train headlamps, and so on. Thermal energy can be obtained from sunlight or the Joule heat of an electric wire accompanying energization. Wind energy can be obtained from natural wind, the wind from passing trains, ventilation wind in tunnels, the wind caused by pressure fluctuations when trains pass through tunnels, and so forth. Vibration energy can be obtained from overhead lines oscillated by natural wind or passing trains, and from the vibration of structures. Magnetic field energy can be obtained from fluctuations in the DC current of the train line. Electric energy can be obtained by combining the current flowing in the train line with the Hall effect. Moreover, it can be obtained from a voltage drop, such as that in an electric wire accompanying a current supply.

\section{Environmental Condition Measurement}

Taking into account the amount of each type of energy and its feasibility among the energy sources in Sect. 2, we measured illuminance, train line temperature, outside temperature, train line vibration, and structure vibration. We also measured the direct current flowing on train lines at a substation to estimate the amount of power generated by the magnetic field, Hall effect, and voltage drop. 


\subsection{Illuminance}

Measurements were made by installing a T-D TR-74Ui illuminance sensor on the roof of Kotari substation between Mukomachi and Nagaokakyo stations on the Tokaido Main Line. The sensor was stored in a waterproof box and installed in a place directly exposed to sunlight. Measurements were performed at 10 min intervals.

Figure 1 shows the average illuminance over $24 \mathrm{~h}$ and the weather for each day. The average illuminance during the measurement period was about $11000 \mathrm{~lx}$, and the average illuminance in the case of cloudy or rainy weather was 4000 to 8000 lx.

\subsection{Vibrations of train lines and structures}

Measurements were made by attaching an accelerometer to a beam between Mukomachi and Nagaokakyo stations and a feeder line supported by it. In addition, we also performed measurements on a beam on an iron bridge between Amagasaki and Tsukaguchi stations on the Fukuchiyama Line and on a bundle suspended from the beam.

Figure 2 shows an example of a waveform measured in the front-rear direction for the bundle suspended from the beam on the Fukuchiyama Line. From the results, it was confirmed that the measurement point vibrates only when a train passes. This was also observed at other measurement points. Figure 3 shows the result of frequency analysis of the waveform in Fig. 2. A maximum acceleration of $4.7 \mathrm{~m} / \mathrm{s}^{2}$ was observed at a frequency of $99 \mathrm{~Hz}$, followed by $3.8 \mathrm{~m} / \mathrm{s}^{2}$ at a frequency of $77.5 \mathrm{~Hz}$.

\subsection{DC current of train lines}

The measurement was carried out by temporarily installing a measuring instrument at the Kyoto substation on the Tokaido Main Line. The measurement line was the outside line in the direction of Yamashina and Nishioji.

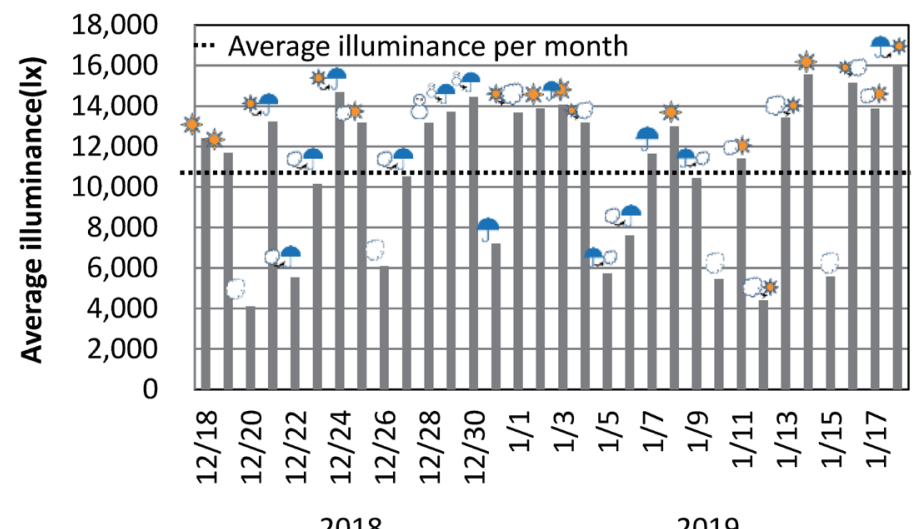

2018

2019

Fig. 1. (Color online) Average illuminance over $24 \mathrm{~h}$. 


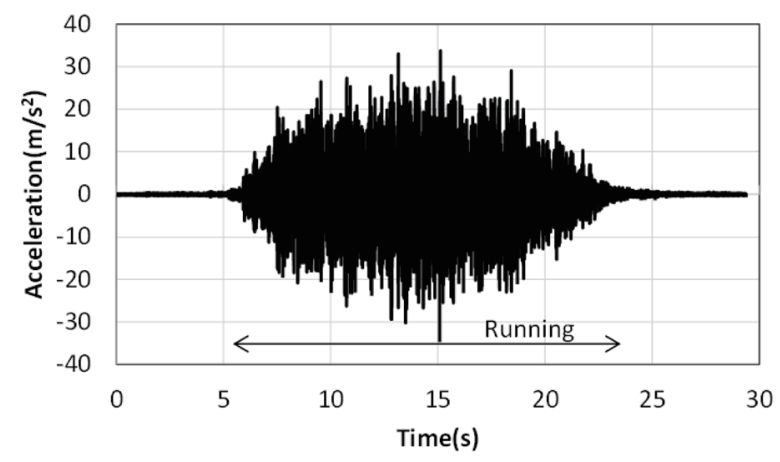

Fig. 2. Acceleration waveform.

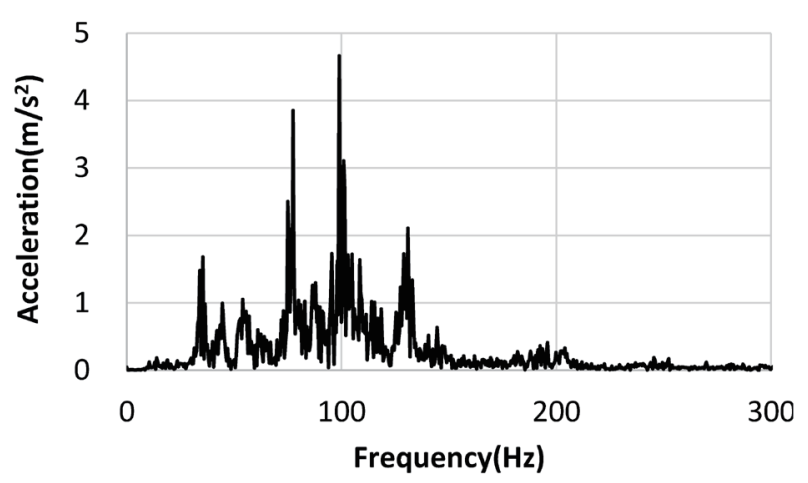

Fig. 3. Frequency analysis result.

The measurement results are shown in Fig. 4. Current exceeding 1000 A was generated intermittently at an interval of around $10 \mathrm{~min}$, with the timing almost coinciding with the times a train departed the station. Periods with a current of $1000 \mathrm{~A}$ or more had a duration of around $1 \mathrm{~min}$. At other times, a current of $500 \mathrm{~A}$ or less was maintained.

\section{Estimated Power Generation}

\subsection{Photovoltaic generation}

Dye-sensitized solar cells were used for the trial calculation because of their good power generation characteristics on cloudy and rainy days. The calculation was made using the characteristics of FDSC-FSC4 $\left(112 \times 56 \mathrm{~mm}^{2}\right)$ and FDSC-FSC6FG $\left(44.5 \times 40.5 \mathrm{~mm}^{2}\right)$ solar cells manufactured by Fujikura. The amount of power generation was calculated from the illuminance results measured every $10 \mathrm{~min}$ in Sect. 3.1 and the characteristics of the illuminance power generation amount of the product. This product is proportional to the illuminance up to $10000 \mathrm{~lx}$ and has a characteristic that becomes a constant value beyond $10000 \mathrm{~lx}$. Therefore, in the case of illuminance of $10000 \mathrm{~lx}$ or more, the illuminance was set to $10000 \mathrm{~lx}$ and was calculated.

Table 1 shows the amount of generated power calculated from the illuminance measurement results obtained in Sect. 3.1. The average amounts of power generated of about $352 \mathrm{~J} / \mathrm{d}$ for the FDSC-FSC4 solar cell and about $67 \mathrm{~J} / \mathrm{d}$ for the FDSC-FSC6FG solar cell were expected. Moreover, even in the case of low illuminance due to bad weather, the amount of power generated of about $50 \mathrm{~J} / \mathrm{d}$ could be obtained with the FDSC-FSC6FG solar cell with a small photovoltaic panel area. The calculation of the amount of power generation is based on seasonal results with low solar radiation. Therefore, it is considered that more power can usually be obtained than the results indicated here. On the other hand, owing to restrictions on the locations of photovoltaic power generation panels, if solar radiation is blocked, it may not be possible to generate the amount of power given here. 


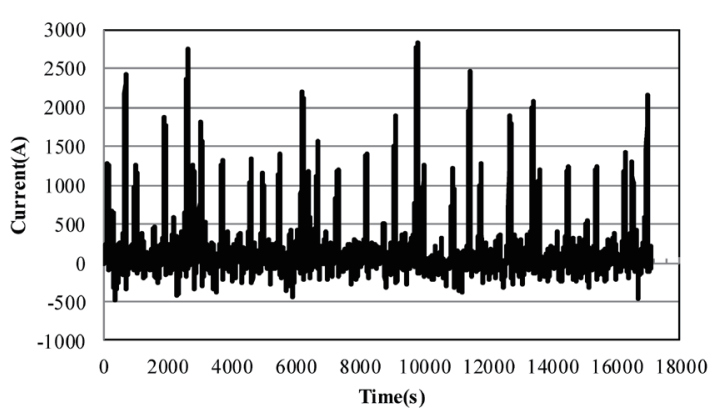

(a)

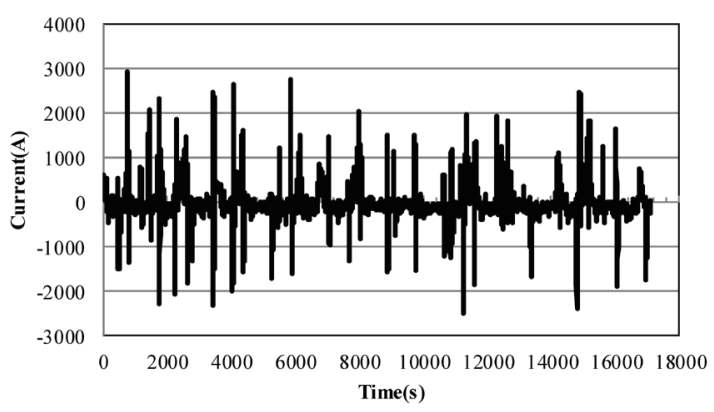

(c)

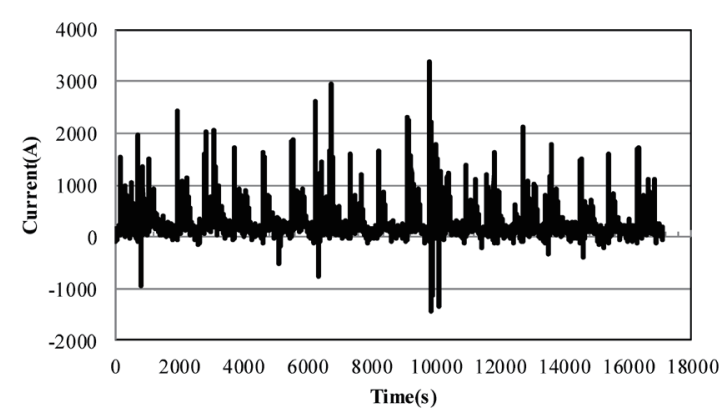

(b)

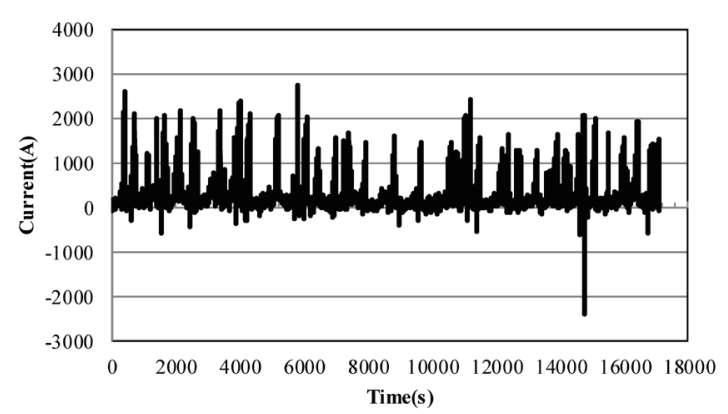

(d)

Fig. 4. DC load current waveforms of (a) A Line, (b) B Line, (c) C Line, and (d) D Line.

Table 1

Photovoltaic power energy.

\begin{tabular}{lcc}
\hline & FDSC-FSC4 (J/d) & FDSC-FSC6FG (J/d) \\
\hline Average electrical energy & About 352 & About 67 \\
Electrical energy on day with lowest illuminance & About 264 & About 50 \\
\hline
\end{tabular}

\subsection{Vibration power generation}

In a trial calculation of the electric energy when using a piezoelectric element with the result obtained in Sect. 3.2, we obtained values of 2.6 and $2.8 \mathrm{~mW}$ at frequencies of 99 and $77.5 \mathrm{~Hz}$, respectively. The characteristics of a piezo power estimator for energy harvesting manufactured by MIDÉ were used for the characteristics of the piezoelectric element. The size of this piezo power estimator is approximately $80 \times 75 \times 20 \mathrm{~mm}^{3}$ and weighs less than $100 \mathrm{~g}$ including the fixed clamp.

Table 2 shows the results of a trial calculation of power generated at other measurement points using the same method and the total amount of power generated per day calculated from the number of passing trains. The number of passing trains per day on the Tokaido Main Line is about 640 and that on the Fukuchiyama Line is about 360. The duration of vibration generated by the passing train was set to $10 \mathrm{~s}$ for the Tokaido Main Line and $15 \mathrm{~s}$ for the Fukuchiyama Line on the basis of actual measurement results. The number of passing trains per day excludes out-of-service and freight trains. 
Table 2

Vibration power generation energy per day.

\begin{tabular}{lcc}
\hline & Point & Energy (J/d) \\
\hline \multirow{2}{*}{ Tokaido Main Line (earth fill) } & Feeder line & 1.92 \\
& Beam & 0.45 \\
\hline \multirow{2}{*}{ Fukuchiyama Line (elevated) } & Beam & 14.04 \\
& Bottom bundle & 15.12 \\
\hline
\end{tabular}

As a result, the energy gained from the vibration of a beam standing on the ground and a feeder supported by it was $2 \mathrm{~J} / \mathrm{d}$ or less. On the other hand, the energy gained from the vibration of the equipment attached to the beam on the bridge was about $15 \mathrm{~J} / \mathrm{d}$. In consideration of the loss of the converter, the actual generated power that can be used by a sensor is considered to be less than this value.

\subsection{Magnetic field power generation}

In a coil installed in parallel with an electric wire, it is possible to obtain a dielectric electromotive force accompanying a change in direct current flowing through the electric wire. Therefore, a coil was installed on a feeder, and the induced electromotive force that can be obtained from the flowing current was calculated. As the conditions of the coil, the number of turns $(N)$ was 100 turns, the area $(S)$ was $0.003 \mathrm{~m}^{2}$, and the average distance from the wire was $0.1 \mathrm{~m}$. The core materials of the coil were SS400, which is easily available, and silicon steel, which can obtain a large magnetic flux density at a low magnetic field strength. The magnetic field strength and magnetic flux density of the materials were based on the characteristics shown in Fig. 5.

It was assumed that a DC1664A boost IC made by Analog Devices was used to obtain the amount of power generated from the electromotive force of the voltage drop. This boost IC operates with an input voltage of $50 \mathrm{mV}$ or more.

Figure 6 shows the waveform of the dielectric electromotive force calculated from the measured data in Fig. 4(a). In this calculation, the voltage drop at which the boost IC does not operate was set to $70 \mathrm{mV}$ with a margin, and the energy generated when the voltage drop is $70 \mathrm{mV}$ or less was set to zero. During the measurement period, the number of times that the induced electromotive force fell below $70 \mathrm{mV}$ was determined to be 245 for the SS400 core and 3203 for the silicon steel core. Since most of the magnetic field generated by train load fluctuations was at currents on the order of 10-100 A, it is considered that the silicon steel generated most of the power. The amount of power that can be generated per day by using a DC/DC converter with an efficiency of $10 \%$ was estimated from the ratio of the number of trains based on the amount of power generated during the time period measured. It was found to be about $0.52 \mathrm{~J} / \mathrm{d}$ for the SS400 core and about $7.53 \mathrm{~J} / \mathrm{d}$ for the silicon steel core. In this calculation, loss of iron is not considered, so the actual amount of power generation is less than this value. 


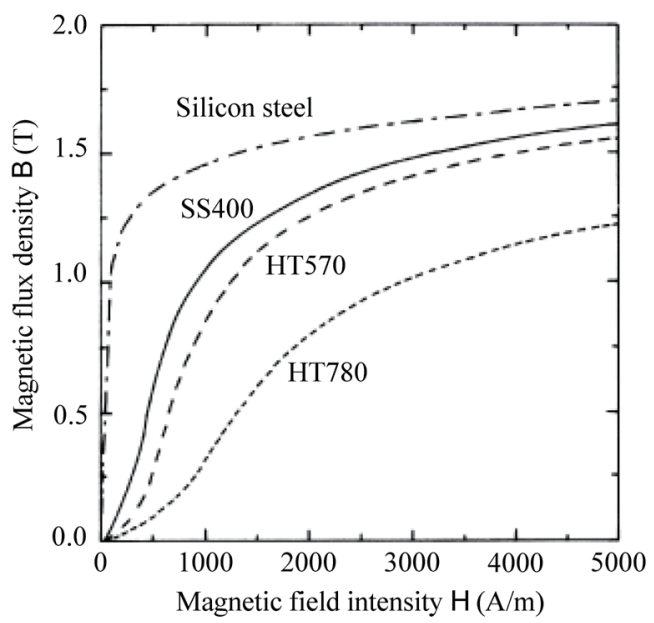

Fig. 5. Characteristics of magnetic field strength and magnetic flux density of materials. ${ }^{(5)}$

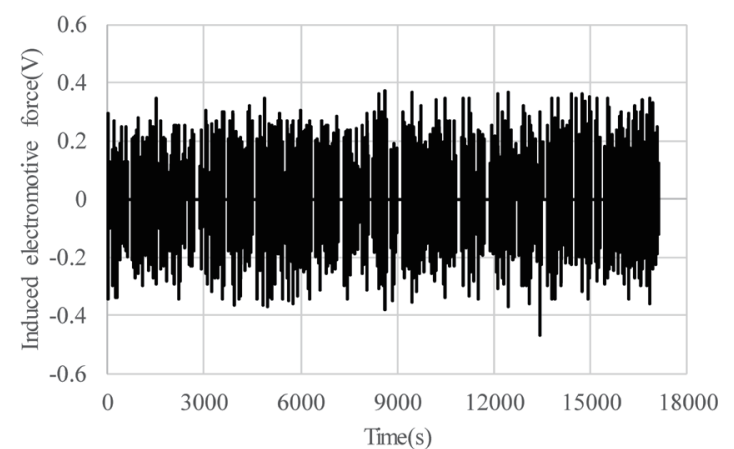

(a)

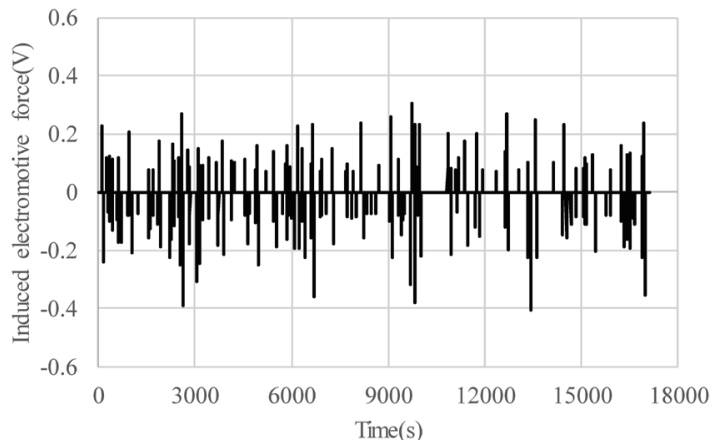

(b)

Fig. 6. Magnetic field power generation energies of (a) silicon steel and (b) SS400.

\subsection{Hall effect power generation}

When a magnetic flux having a magnetic flux density $(B)$ T perpendicular to a current $(I)$ A is applied, a Hall electromotive force $(V) \mathrm{V}$ is generated. Therefore, an electromotive force can be obtained from the load current of a train by attaching a magnet to the feeder. The Hall electromotive force $(V) \mathrm{V}$ can be expressed by

$$
V=R \times B \times I / a,
$$

where $(R) \mathrm{m}^{3} / \mathrm{C}$ is the Hall coefficient of the object and $(a) \mathrm{m}$ is the thickness of the object in the direction orthogonal to the magnetic flux.

The electromotive force when a DC current of 1000 A flows through an aluminum feeder with a diameter of $30 \mathrm{~mm}$ to which a magnet with a magnetic flux density of $1 \mathrm{~T}$ is attached is $1.03 \times 10^{-6} \mathrm{~V}$ from Eq. (1). The Hall coefficient of aluminum is $3.1 \times 10^{-11} \mathrm{~m}^{3} / \mathrm{C}$. Therefore, the electromotive force that can be obtained even at higher currents is on the order of $\mu \mathrm{V}$. 


\subsection{Voltage drop power generation}

In a sensor installed between two points on an electric wire, an electromotive force can be obtained by a voltage drop caused by energization. We calculated the amount of electric power that can be generated by the voltage drop that arises in the compression connection part of a feeder. In the calculation, we assumed that a compression sleeve having a length of $500 \mathrm{~mm}$ is attached to the center of a $510 \mathrm{~mm}^{2}$ aluminum wire having a total length of $1 \mathrm{~m}$. The crosssectional area of the compression sleeve is $144 \mathrm{~mm}^{2}$, each resistance feeder has a resistivity of $2.89 \times 10^{-8} \Omega \cdot \mathrm{m}$, and the compression sleeve has a resistivity of $2.65 \times 10^{-8} \Omega \cdot \mathrm{m}$. At this time, the combined resistance of the feeder cable including the compression sleeve, ignoring the contact resistance between the sensor and the cable, is about $35 \mu \Omega$. In addition, since the resistance of the compression sleeve increases to $100 \mu \Omega$ or more as it deteriorates, the combined resistance in this case is assumed to be about $350 \mu \Omega{ }^{(6)}$

It was assumed that a LTC3108 boost IC made by Analog Devices was used to obtain the amount of power generated from the electromotive force of the voltage drop. This boost IC operates with an input voltage of $20 \mathrm{mV}$ or more. The amount of power generated was calculated from the current obtained by actual measurement in Fig. 4 and the combined resistance of the compression part.

Figure 7 shows an example of the estimated waveform of the amount of power generated by a normal compression sleeve. In addition, Table 3 shows the results of calculating the amount

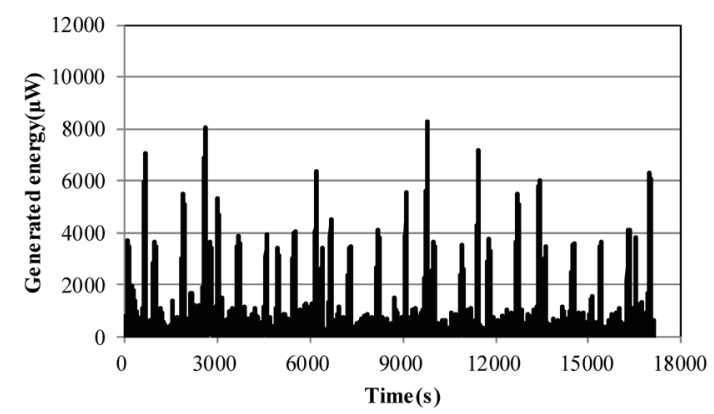

(a)

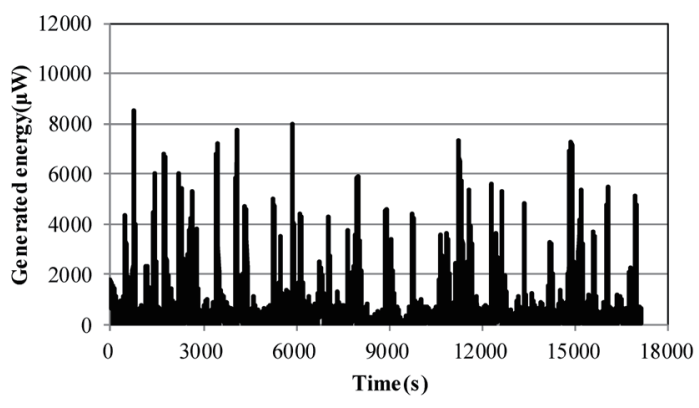

(c)

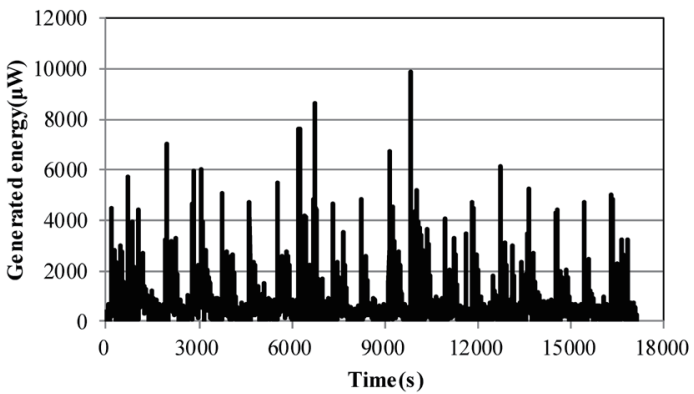

(b)

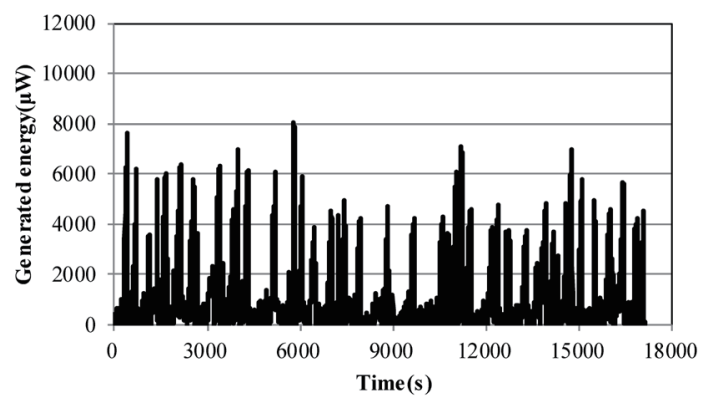

(d)

Fig. 7. Waveforms of voltage drop power generation energies of (a) A Line, (b) B Line, (c) C Line, and (d) D Line (normal compression). 
Table 3

Voltage drop power generation energy.

\begin{tabular}{lccccc}
\hline & A Line $(\mathrm{J} / \mathrm{d})$ & B Line $(\mathrm{J} / \mathrm{d})$ & C Line $(\mathrm{J} / \mathrm{d})$ & D Line $(\mathrm{J} / \mathrm{d})$ & Average $(\mathrm{J} / \mathrm{d})$ \\
\hline Normal compression sleeve & 0.76 & 0.61 & 0.71 & 1.12 & 0.80 \\
Deteriorated compression sleeve & 40.30 & 47.05 & 45.07 & 59.19 & 47.90 \\
\hline
\end{tabular}

of power generated per day on the basis of the ratio of the number of trains to the amount of power generated during the measurement time period. In this calculation, the amount of power generated when the voltage drop at which the boost IC dose not operate was $20 \mathrm{mV}$ or less was set to zero. As a result, the amount of power generated was about 0.6 to $1.1 \mathrm{~J} / \mathrm{d}$ for a normal compression sleeve and about 40 to $60 \mathrm{~J} / \mathrm{d}$ for a deteriorated compression sleeve.

\section{Examination of Application to Wireless Sensor Power Supply}

We verified and evaluated the applicability of the electric energy calculated from the measured environmental energy as a wireless sensor power supply.

\subsection{Sensor power consumption}

The sensor used for verification performed measurements at predetermined time intervals, transmitted data after each measurement, and was inactive at other times. The measurements and data transmission were verified in five patterns of every $1 \mathrm{~min}, 10 \mathrm{~min}, 30 \mathrm{~min}, 1 \mathrm{~h}$, and 24 h. It was assumed that $50 \mu \mathrm{W}$ is consumed for measurement, $10 \mu \mathrm{W}, 100 \mu \mathrm{W}, 1 \mathrm{~mW}$, or $10 \mathrm{~mW}$ is consumed for communication, and $33 \mu \mathrm{W}$ is consumed for standby. It was assumed that the measurement takes $1 \mathrm{~s}$ and the communication takes $9 \mathrm{~s}$. The efficiency of each device was assumed to be $50 \%$.

Figure 8 shows the results of calculating the power consumption per day from these conditions. From the results, it can be seen that the more frequently the measurement and communication are performed, the greater the effect of the power consumed for communication. If the measurement and communication are performed once per day, the effect of the power consumed for communication is almost eliminated. On the other hand, when the power consumed for communication is $10 \mathrm{~mW}$, power consumption of 10 to $100 \mathrm{~J} / \mathrm{d}$ order is required when measurement and communication are performed once per hour. In addition, when the power consumed for communication is $1 \mathrm{~mW}$ and the measurement and communication are performed at intervals of $1 \mathrm{~min}$, energy on the order of $10 \mathrm{~J} / \mathrm{d}$ is required.

\subsection{Consideration}

The application of different methods of power generation to a wireless sensor power supply will be studied on the basis of the calculated amount of power generated. Since solar power generation can obtain energy on the order of $10 \mathrm{~J} / \mathrm{d}$, it is considered that it can be applied under conditions where the power consumed for communication is $1 \mathrm{~mW}$ or less. In addition, it can 


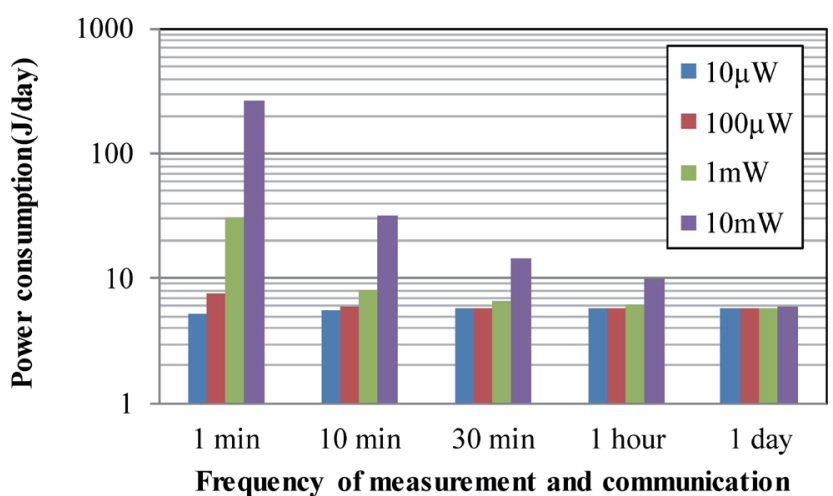

Fig. 8. (Color online) Sensor power consumption.

be applied if the frequency of measurement and communication is set to $10 \mathrm{~min}$ or longer even when the power consumed for communication is $10 \mathrm{~mW}$. It is considered that vibration power generation can be applied upon suppressing the power consumed for communication or the frequency of communication and measurement under a condition with vibration such as on an elevated beam. Magnetic field power generation can be applied by suppressing the power consumed for communication or the frequency of communication and measurement, depending on the train current and fluctuation frequency. Voltage drop power generation can be applied when the compression sleeve is deteriorated but is difficult to apply when it is undamaged. For this reason, it is considered that this power generation method can be applied in operations where communication is performed when deterioration progresses rather than in the case of continuous monitoring.

It is conceivable that the data is collected from a traveling train, an adjacent station, a substation, or an office. The distance from the sensor can be assumed to be less than $10 \mathrm{~m}$ for a traveling train, less than $10 \mathrm{~km}$ for a station or substation, and more than $10 \mathrm{~km}$ for an office. When collecting data from a traveling train, it is considered that high-frequency communication is possible with low power consumption for communication because the communication distance is short. However, depending on the route, the frequency of trains passing is from several minutes to several hours, and the frequency of communication depends on this frequency. On the other hand, for stations, substations, and offices, frequent communication is considered undesirable because communication with high power consumption is required owing to the long communication distance.

\section{Conclusions}

Assuming that a wireless sensor is installed to determine the state of facilities along a railway, different types of environmental energy as a possible power source for the sensor were measured, and the amount of electric power thus obtained was estimated. The possibility of applying each type of environmental energy as a power source of a wireless sensor was evaluated from the results of the trial calculation. In addition to solar power generation, where 
the largest amount of power generated was expected, other power generation methods yielded results that can be applied depending on the installation location, the frequency of measurement and communication, and the data collection method used. Furthermore, by combining two or more power generation methods or combining them with secondary batteries, the frequency of measurement and communication and the communication distance can be increased.

\section{References}

1 S. Yamada, T. Tsumori, and S. Honda: 2016 IEEJ Industry Appl. Soc. Conf. No. 5-24 (2016) 231 (in Japanese).

2 W. Nakashima, T. Wakabayashi, Y. Sugama, and A. Yanase: 2016 IEE Japan Conf. 5-184 (2016) 289 (in Japanese).

3 H. Waki, N. Ishioka, and T. Nagashima: Papers of Technical Meeting on Transportation and Electric Railways, IEEJ, TER-11-018 (2011) (in Japanese).

4 T. Sugiyama, Y. Shibata, G. Hashiguchi, S. Takeuchi, H. Tanaka, K. Kawahara, H. Koga, and H. Mitsuya: 2018 IEEJ Industry Appl. Soc. Conf. 5-26 (2018) 257 (in Japanese).

5 The Japan Welding Engineering Society: http://www-it.jwes.or.jp/qa/details.jsp?pg_no=0030030100 (accessed September 2019).

6 A. Nozue, T. Nakazawa, E. Nishida, and T. Saito: AEW 30 (2001) 11 (in Japanese). 